\title{
An Intellectual Journey in History: Preserving Indian Cultural Heritage
}

\author{
Anupama Mallik ${ }^{1}$, Santanu Chaudhury ${ }^{1}$, T.B. Dinesh ${ }^{2}$, and Chaluvaraju ${ }^{3}$ \\ 1 Indian Institute of Technology, Delhi \\ \{ansimal, chaluvaraju.hampi\}@gmail.com, santanuc@ee.iitd.ac.in \\ 2 International Institute for Art, Culture and Democracy, Bengaluru, India \\ dinesh@iiacd.org \\ 3 Kannada University, Hampi, India
}

\begin{abstract}
Heritage preservation requires preserving the tangibles (monuments, sculpture, coinage, etc) and the intangibles (history, traditions, stories, dance, etc). Besides these artefacts, there is a huge amount of background knowledge that correlates all these resources and establishes their context. In this work, we present a new paradigm for heritage preservation - 'an Intellectual Journey into the past', which is more advanced than physical explorations of heritage sites and virtual explorations of monuments and museums. This paradigm proposes an experiential expedition into a historical era by using an ontology to inter-link the digital heritage artefacts with their background knowledge. A multimedia ontology encoded in the Multimedia Web Ontology Language (MOWL) is used to illustrate this paradigm by correlating the digital artefacts with their history as well their living context in today's world. The user experience of this paradigm involves a virtual traversal of a heritage site, with an ontology guided navigation through space and time and a dynamic display of different kinds of media.
\end{abstract}

\section{Introduction}

Heritage is not just an accumulation of tangible artefacts and intangible knowledge, preserved by presenting these resources to people to view, explore and analyse. To understand heritage, one must take a journey down several paths in history and experience the various events in their historical and cultural context. This journey can be carried out in space and time with the help of technology. It can be augmented by intellectual inputs from a collection of beliefs, customs and knowledge, and audio-visual inputs of digitized resources, placed in context and correlated by an ontology. In this work 1 , we propose a new research paradigm in the domain of cultural heritage preservation, which entails an intellectual journey of an era, allowing a person to explore a heritage site virtually and experience the heritage in its past and current context.

${ }^{1}$ This research work has been funded by the heritage project entitled "Managing Intangible Cultural Assets through Ontological Interlinking" of the Department of Science and Technology of the Government of India.

A. Petrosino, L. Maddalena, P. Pala (Eds.): ICIAP 2013 Workshops, LNCS 8158, pp. 298-307, 2013.

(C) Springer-Verlag Berlin Heidelberg 2013 
While exploring a heritage site, what if one has immediate access to all the information about every monument, sculpture, art or mural painting that one looks at; information which is audio-visual and dynamic. Imagine being able to view the video of a dance performance portraying the dance posture depicted in a sculpture, or read the text of a narrative portrayed in a mural painting on a temple ceiling; or compare with images of other mural paintings at other sites, depicting the same narrative. What if besides hearing a recorded history of a monument on an audio-guide, one could watch videos of rituals which take place at the same site in current times, as well as hear recordings of different views given by various experts. This kind of experiential exploration is proposed in our paradigm which we illustrate with the help of an interactive user interface described in this paper. The intellectual journey that we propose here, is much more informative and advanced than physical exploration of heritage sites or the walk-throughs and virtual tours currently offered by online museums and websites [2, [6]. Other related works in cultural heritage preservation like [5] make use of augmented and virtual reality, but fail to present artefacts in context with background knowledge. A similar ontology-based framework for the digital exploration of cultural heritage objects has been used in [1], but authors have not looked at traversal over time possible through correlation of classical and historical narratives with living traditions and folk-lore.

The rest of the paper is organized as follows: Section 2 discusses Intellectual Journey paradigm, and details the architecture of the ontology based framework. In section 3, we discuss the advantage of using a MOWL encoded multimedia ontology in a heritage domain. Section 4 details the ontology-based annotation of the digital artefacts which is required for providing their semantic inter-linkages. Section 5 gives an overview of the ontology based interlinking of digital artefacts. In section 6 we illustrate our paradigm and framework with a web-based application in the domain of Indian Heritage. In section 7, we conclude our findings and discuss future work.

\section{Architecture of Ontology Based Intellectual Journey}

In this section, we discuss the proposed ontology-based heritage preservation scheme, offering an Intellectual journey into history, which correlates and presents various aspects of cultural heritage linked with the user context. Figure $1 \mathrm{~b}$ shows the space and time traversal of heritage resources, offered by our ontology-based framework. The tangibles and intangibles in cultural heritage can belong to different eras, but can broadly be classified into belonging to the Present and Past times. Digital archiving of the various artefacts is done in different ways and the outputs are multimedia collections in different modalities, with different modes of perusal, as shown here:

1. Visualization of present day tangibles - monuments, coins, sculpture, inscriptions as they exist today.

2. Digitization of present day intangibles - recording of live events like festivals, craft traditions, dance and music, as they happen in current times. 


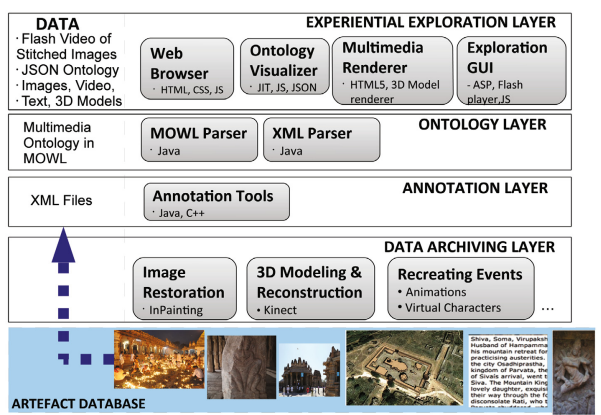

(a) Software Architecture

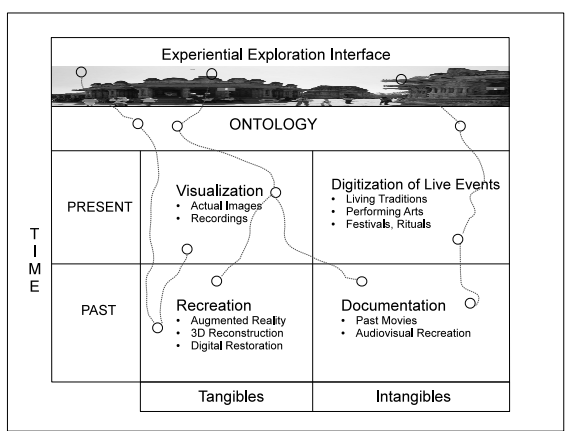

(b) Space-Time Exploration

Fig. 1. Ontology-based Intellectual journey paradigm

3. Recreation of the tangibles, as they existed in the past. This utilises techniques such as 3D modelling, augmented reality, visual reconstruction and image restoration for reconstructing the tangibles as they were, before their temporal degeneration.

4. Documentation of the intangibles. This involves documenting events of the past by collecting facts from recorded history like scriptures, and through making of historical movies, documentaries, etc. that recreate the images and events of an era.

Figure 1a shows the software architecture of the framework that offers the intellectual exploration experience using an ontology. The different layers in this architecture are:

Digital Archiving Layer: This layer consists of all the technical modules for capturing and recreating the digital artefacts. The output of this layer are digital collections of the artefacts of different modalities, i.e. image, text, 3D, video and audio collections.

Annotation Layer: It consists of annotation tools required for annotating and labelling the documents in artefact collections. This step assumes that a multimedia ontology for the domain has been acquired and semantic labels associated with domain concepts in the ontology are available for labelling the artefacts. Depending on the type of document (image, audio, video, text), different tools are required to identify artefacts within that document, For e.g. image cropping, selection, demarcating video segments, identifying individual video frames, etc. Semantic annotation involves labelling each artefact with one or more ontology concepts. This layer produces an MPEG-7 based XML file per document in the collections, with annotations for multiple artefacts in the document.

Ontology Layer: This layer consists of the language parsers - for the ontology as well as for the XML files. The ontology is parsed and web-compatible visualization graph is produced. The XML files are parsed to produce sets/chains of artefacts which are linked through common ontology concepts. Thus the ontology not only correlates the concepts through domain knowledge, it also produces data linkages which serve the purpose of an ontology-based exploration and cross-modal access of media. 
Experiential Exploration Layer: This layer is composed of web-based graphic user interfaces for exploring a heritage site, for ontology visualization and for presenting or rendering the digital artefacts. The user interfaces with this layer to carry out an Intellectual Journey as proposed by our paradigm.

\subsection{Space and Time Traversal}

The Experiential exploration interface allows a complete space traversal of a heritage site, with entry points leading to the interior of different monuments, and option to exit and return to a central location, as desired by the user. Thus the user can move along several spatial paths in the site, as per her choice. This space traversal is augmented by a time-traversal in history, with the help of an interactive interface. Clickable icons placed on tangible artefacts visible in the structure, allow the user to access digital media related to the artefact. This access occurs through data linkages provided by an underlying ontology. The user-click maps to a concept node in the ontology graph. The XML files with artefact annotations are searched to produce the set of digital artefacts which have their semantic annotation matching this concept. The various image, video, $3 \mathrm{D}$ and text rendering modules are triggered to present these artefacts as a correlated set to the user as part of the exploration interface. Besides the space and time traversal in the heritage experience, our system also enables a perusal of the heritage resources on demand by the user, through interactions with the ontology, which allow selection of concepts and/or their relations and attributes.

A user of this system can explore a heritage site virtually, recreated using actual site images. Here are two examples of the different paths she can take in space and time with ontology-guided navigation. Path1: User clicks on a temple icon on screen. It maps to a concept in the ontology. XML annotation files are searched for annotations pertaining to this concept. This search produces a set of images of the temple as it exists now, a text detailing its history and a video of a tour of its premises, and a 3D model of the temple as it was built originally. These are presented to the user. Path2: User views the video of a weekly market or Bazaar (present intangible) as it happens at a temple site today. Then she selects a time-period (15th century) in history through the ontology. The system retrieves an animation movie (past intangible) which recreates the Bazaar as it used to happen in the 15 th century in that temple. Figure $1 \mathrm{~b}$ shows these paths as threads.

\section{Multimedia Ontology and Its Representation}

An ontology encoded in a traditional ontology language, e.g. OWL, uses text to express the domain concepts and their properties, and thus can be used for semantic text processing. Semantic processing of multimedia data, however, calls for ontology primitives that enable modeling of domain concepts with their observable media properties. This kind of modeling is called Perceptual Modeling, which needs to encode the inherent uncertainties associated with 
media properties of concepts as well. Traditional ontology languages do not support these capabilities. We have used the Multimedia Web Ontology Language (MOWL) 4] for representing the multimedia ontology used in our heritage experiments. In [7, authors had proposed an ontology-based scheme based on MOWL, for preserving the intangible heritage of Indian classical dance. Techniques of annotation and concept recognition detailed in [7] have been reused here, but the interlinking of heritage artefacts across media formats using MPEG-7 based metadata, and the experiential exploration and browsing interface for historical narratives is a novel paradigm that we have introduced in this paper.

A multimedia ontology helps encode media properties of the semantic concepts. For our illustration, we have used a MOWL encoded Indian heritage ontology to associate media properties of the multimedia segments with ontology concepts. As MOWL representation allows collection-independent modelling of the domain, different kinds of media - images, text, video and audio can also be associated with the concepts, which aid in perceptual modelling of the concepts. This also helps in building semantic conceptual linkages between different modalities, and can be used to provide inter-linking of the digital artefacts which are part of the heritage collection.

\section{Artefact Annotation}

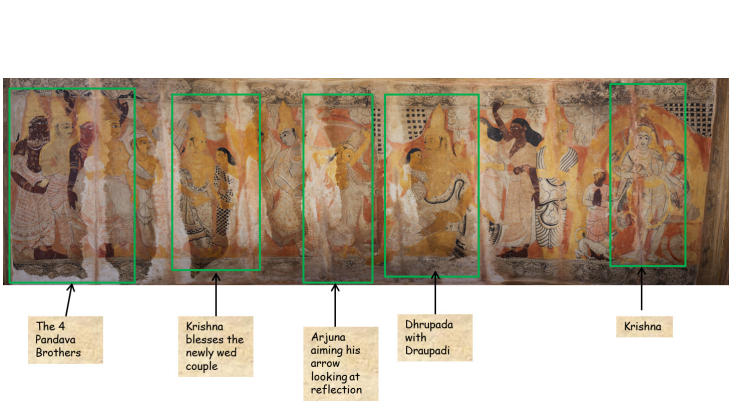

(a) Tagging of a Mural.

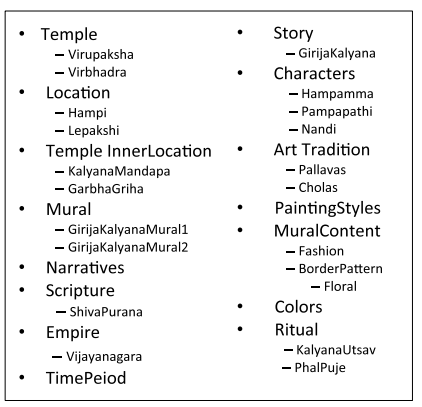

(b) Tag Dictionary.

Fig. 2. Annotation of GirijaKalyanaMural

Domain knowledge derived from experts and other sources, is used to create a tag dictionary which contains different tags that can be used for labelling different aspects of the artefacts. A sample of such a tag dictionary is shown in figure 2b, Typically the tag dictionary contains abstract domain concepts, some of which for a heritage domain are story, narrative, scripture, characters, rituals, location, temple, time period, technique, artistic style, color, etc. The tag dictionary when used for actual tagging of the artefacts, contains the data instances of these concepts - i.e. names of the characters, temples, rituals, scriptures, patterns, art style, colors used, etc. 
An ontology expert analyses the tag dictionary, and generates an ontology of the domain. The ontology is enriched with media data from the collections as media segments are associated with appropriate semantic concepts in the ontology. This process is semi-automated, as it utilises machine learning of the associations between media content and semantic concepts, to generate automatic labels for media segments, but needs to be curated by a domain expert. Using the tag dictionary, the domain experts also provide tags and labels for different segments of the heritage collection. The digital artefacts belong to different media collections of text, audio, images and videos. The tag dictionary is used as the basis for providing tags for image segments, video shots, audio tracks and text segments in the digital collection. An example of tagging of a mural painting is shown in figure 2a, Different annotation tools used for tagging of different kinds of media, produce XML annotation files in a standard format which is based on MPEG-7 based media descriptions.

\section{Ontology Based Interlinking of Digital Artefacts}

In this section, we show how the multimedia ontology, by its collectionindependent modelling of the domain, provides a robust basis for a semantic inter-linking of the digital artefacts collected in repositories stored in different media formats. XML files produced from the annotation of artefacts contain semantic labels linked with media segment descriptions. Thus media segments with same conceptual labels can be hyper-linked for access as required in retrieval through textual search queries or through a graphic user interface which provides an image or icon to click for retrieval of associated multimedia documents. The algorithm which shows how retrieval of media from different media collections is made possible through ontology-based interlinking is shown in algorithm 1.

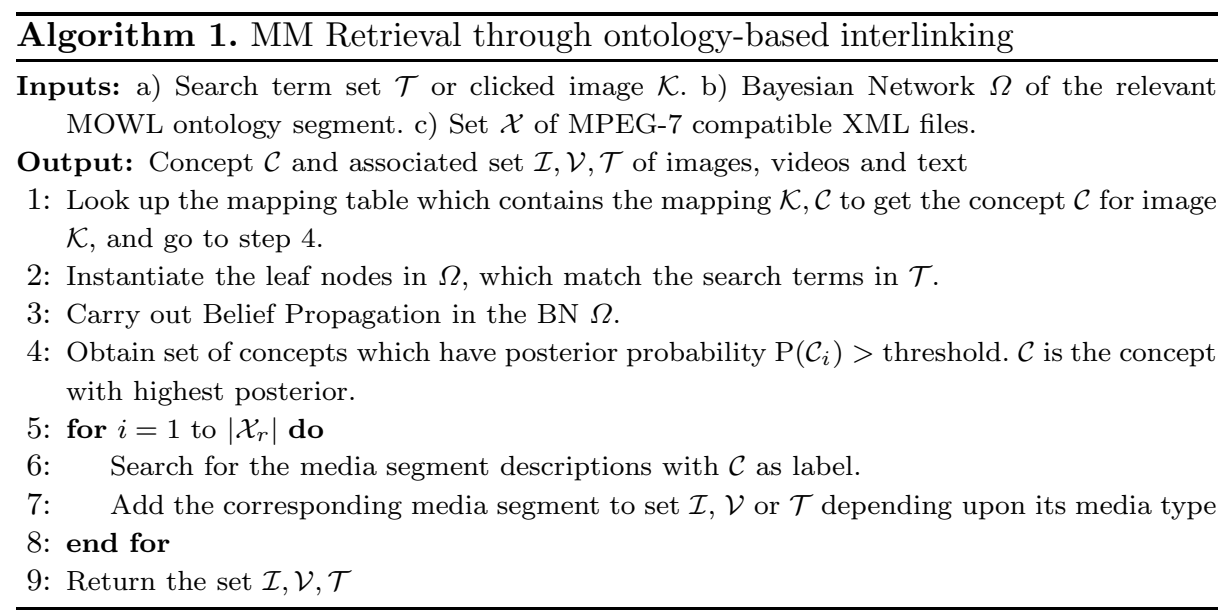




\section{Intellectual Journey in Indian Heritage Domain}

To demonstrate the Intellectual Journey paradigm, using our ontology-based framework, we have chosen the Indian heritage domain related to the UNESCO World heritage site of Hampi, in Karnataka, India. The framework is generic and extensible to preserve any heritage site in the world for which background knowledge and heritage resources are available. Hampi site comprises of the ruins of Vijayanagara city, former capital of the powerful Vijayanagara Empire, which flourished in South India during 14th to 17th century. Several dynasties which ruled during this period, patronised art and culture, built several new temples and renovated and enlarged many old ones. Festivals and rituals involving community participation were encouraged, and in fact still take place at the site.

We further focus on the theme of Girijakalyana from our knowledge-base of the Hampi heritage. GirijaKalyana refers to the marriage between goddess Hampamma (also known as Uma, Parvati or Shakti) and male Hindu god Pampapathi (also known as Shiva, Shankara or Rudra). The concept manifests in both tangible and intangibles of Hampi as well as some other Vijayanagara sites like Lepakshi in Andhra Pardesh, India. The tangible manifestations include mural paintings depicting the marriage on the walls of ancient temples in Hampi and Lepakshi; sculptures and bronzes characters in the story, found in temples and museums, and so on. There is a story attached to the concept, text of which is found in certain ancient scriptures like the ShivaPurana. Certain aspects of this mythological story are also found in narratives in old inscriptions, memorial stones and manuscripts. Narratives of some performing arts like folk dance, theatre and puppetry in areas around Hampi and Lepakshi abound in references to the marriage of Shiva and Parvati.

GirijaKalyana context has a living heritage. Every year the marriage is celebrated in the ancient temple of Hampi. Communities from nearby areas congregate to attend the two famous rituals of Kalyanautsava (the wedding), preceeded by Phalpuje (the engagement). Priests actually conduct a wedding of the deities in the temple, and people make offerings to please the Gods. Traditional crafts like making puppets, toys, wooden door-frames and wall-paintings are still

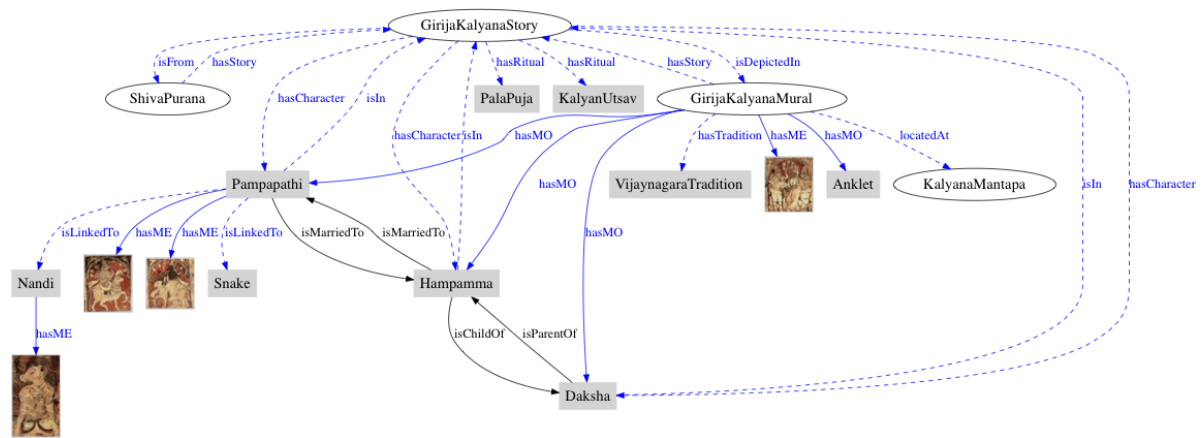

Fig. 3. Indian heritage ontology snippet with focus on GirijaKalyana concept 
being practised in locations around Hampi. Many of these replicate images and patterns from the Girijakalyana concept. It is a fashion in local communities to get tattos depicting some GirijaKalyana patterns drawn on their person.

\subsection{Resource Compilation}

For our demo, we collected digitized versions of the tangible artefacts such as mural paintings, sculptures, crafts and manuscripts, related to GirijaKalyana, as well as audio-visual recordings of the intangibles such as the story, craft traditions, rituals and folk narratives. After segmenting, labelling and tagging of the images, there were 654 image segments. The other repository is of 126 stories from Indian mythology and folk-lore, with 3 different versions of each story on an average. The text segments after labelling and tagging for different characters, episodes, locations, etc. are approximately 1000. Our cultural teams were able to collect about 12 hours of video recordings of the various site visits, ritual celebrations and craft traditions. These media files were segmented into 95 video shots of varying length, which were then tagged and annotated with domain concepts in the ontology, as detailed in section 4 .

Background knowledge about the concept was provided to the ontology expert by our group of domain experts - mainly art and cultural historians who specialise in the study of the Vijayanagara empire with a focus on the Hampi world heritage. We also collected facts and resources from books [8] written about Hampi and its art and archaeology. This knowledge along with the tag dictionary was then used in creating the Indian heritage ontology, which has approximately 156 concepts, 83 of which have media examples and patterns associated with them. Our cultural and ontology teams undertook 3 months of discussion, validations and corrections to produce the final version of the ontology, and 4 months to label and tag the artefacts with the semantic concepts. Part of annotation was semi-automatic as discussed in section 4, which was then curated by the experts.

A multimedia ontology snippet of the GirijaKalyana context, after its creation from the tag-dictionary in figure 2b, is shown in the figure 3. We focus on the GirijaKalyanaStory with characters Hampamma and Pampapathi, with its depictions in mural paintings, rituals and current traditions. A Mural painting depicting the GirijaKalyanaStory also has some other content besides the depiction of the story or narrative, like floral or geometric patterns, some fashion patterns like hair-styles and jewellery.

\subsection{Experiential Exploration Interface}

Once the annotated repositories are available, and the multimedia ontology has been generated, we allow the user to virtually explore a heritage site, using an interactive graphic user interface, which has an ontology-guided navigation and a dynamic display of associated digital artefacts. This is an intellectual journey around the GirijaKalyana manifestation in Hampi heritage. User experience of this journey involves a traversal of the stitched image sequence of the Hampi 


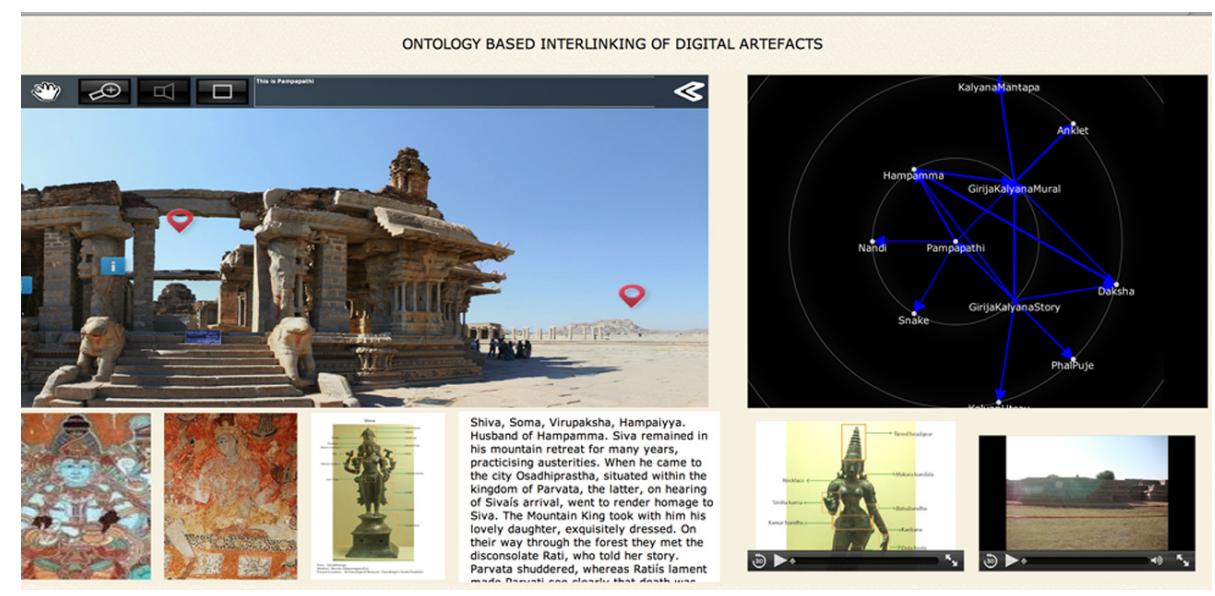

Fig. 4. Screen shot with Pampapathi concept selected

heritage site with a window view of the related ontology snippet as a frame of reference. Navigation is ontology guided where socio-cultural context of the frame is portrayed through a dynamic display of media and narratives.

Figures 4 is a screen shot of our GUI, which has a main pane, showing a 360 degree photo-realistic stereo view of the heritage site, created by stitching of actual images of the site. The user is allowed to explore the site virtually, with intelligent interactions, using mouse buttons to move left, right and other navigation controls for zooming and auto-rotating. The right pane shows an animated graphic view of the ontology, with a node in the center, which reflects the current context of the user selection. This visualization of the ontology graph is created by using the weighted graph animation tools of the Javascript Infovis Toolkit [3], with a JSON structure of the ontology. The frames at the bottom display images, text and videos associated with the concept node at the center of the ontology. If another concept node is selected by clicking on it in the ontology pane, the visualization perspective changes to center this node.

While moving around virtually, the user has an option to click on certain visual cues in order to alter a visual display of image, text and videos of the artefacts as well as change the perspective of the ontology visualization shown in a parallel window on screen. As the user context changes dynamically, so does the display of artefacts and the view of the ontology. User also has an option to navigate to a chosen concept in the ontology window by clicking on it. This too alters the set of artefacts displayed on screen. User evaluation of this interface is planned in near future and is not part of this work.

\section{Conclusion}

Physical or virtual tours of heritage sites need to be augmented with readily available contextual information to the tourist. Mere perusal of audio guides and 
visual cues is not sufficient to completely satisfy the information need. In this paper, we have proposed a new paradigm for a exploring heritage. The proposed paradigm offers an Intellectual journey into a heritage site, with readily available tools to traverse in space and time and access e-Heritage artefacts in context and on demand. We have illustrated this paradigm with a graphic user interface which offers ontology-guided navigation and dynamic display of artefacts in context of the theme of GirijaKalyana related to the World heritage site of Hampi in India. Such an ontology-based framework can easily be extended to offer cross-modal access to heritage resources preserved in different digital media formats.

\section{References}

1. Aliaga, D.G., Bertino, E., Valtolina, S.: Decho - a framework for the digital exploration of cultural heritage objects. J. Comput. Cult. Herit. 3, 12:1-12:26 (2011), http://doi.acm.org/10.1145/1921614.1921619

2. ASI-India: Archaeological survey of india - home, http://www.asi.nic.in/index.asp

3. Belmonte, N.G.: Javascript infovis toolkit, http://philogb.github.io/jit/index.html

4. Ghosh, H., Chaudhury, S., Kashyap, K., Maiti, B.: Ontology Specification and Integration for Multimedia Applications. In: Ontologies: A Handbok of Principles, Concepts, and Applications in Information Systems. Springer (2007)

5. Ikeuchi, K., Oishi, T., Kagesawa, M., Banno, A., Kawakami, R., Kakuta, T., Okamoto, Y., Lu, B.V.: Outdoor gallery and its photometric issues. In: Proceedings of the 9th ACM SIGGRAPH Conference on Virtual-Reality Continuum and its Applications in Industry, VRCAI 2010, pp. 361-364. ACM, New York (2010), http://doi.acm.org/10.1145/1900179.1900254

6. Louvre: Museum website, http://www.louvre.fr/llv/commun/home.jsp?bmLocale=en

7. Mallik, A., Chaudhury, S., Ghosh, H.: Nrityakosha: Preserving the intangible heritage of indian classical dance. JOCCH 4(3), 11 (2011)

8. Verghese, A., Dallapiccola, A.L. (eds.): South India under Vijayanagara: Art and Archaeology. Oxford University Press (2011) 